\title{
Aprendizaje de la comunicación oral en lengua alemana con apoyo de las TIC \\ Using ICT to support Learning of Oral Communication in German Language
}

\author{
José-V. Benlloch-Dualde ${ }^{1}$, Daniela Gil-Salom² ${ }^{2}$ Coral López-Mateo ${ }^{2}$ \\ jbenlloc@disca.upv.es, dagil@idm.upv.es, clopezm@idm.upv.es \\ ${ }^{1}$ DISCA-ETSINF \\ Universitat Politècnica de València \\ Valencia, España \\ ${ }^{2}$ Departamento de Lingüística Aplicada \\ Universitat Politècnica de València \\ Valencia, España
}

\begin{abstract}
Resumen- Uno de los objetivos específicos de este proyecto es la creación de recursos educativos digitales en soporte vídeo, para optimizar el aprendizaje de la comunicación oral formal en lengua alemana. Los alumnos matriculados en las asignaturas de Alemán en diferentes grados de la Universitat Politècnica de València, aprenden a realizar presentaciones orales como parte de la competencia comunicativa para su futuro profesional y/o académico. La innovación consiste en la creación de vídeos interactivos a partir de las mismas presentaciones de los alumnos, con el objeto de facilitar una guía de buenas prácticas. De esta manera se espera que aumente la motivación del alumnado al poderse desarrollar un aprendizaje inter pares. Por otro lado, para desarrollar una evaluación formativa adecuada, la autoevaluación es indispensable y para que esta última sea fructífera, es altamente recomendable que el alumno pueda visionar sus propias presentaciones. De ahí la conveniencia de combinar ambas estrategias. Como trabajo futuro, se efectuará un análisis en el que se procesarán los datos correspondientes al uso de recursos didácticos en la plataforma institucional, junto a los datos de los logros de aprendizaje globales. Todo ello junto a la valoración del proyecto por parte del alumnado y del profesorado.
\end{abstract}

Palabras clave: alemán como lengua extranjera, comunicación oral efectiva, evaluación formativa, vídeos interactivos.

Abstract- One of the specific aims of this project is to create digital learning resources in video format, in order to optimize the learning process of formal oral communication in German. The students enrolled in the subject German at different Schools of the Universitat Politècnica de València learn to make oral presentations as part of the communication skill for their professional and/or academic future. The innovation consists in creating interactive videos based on the same students' presentations, in order to facilitate a good practice guide. In this way, through the development of peer-learning, an increase of the student's motivation is expected. Additionally, to carry out an appropriate formative evaluation, self-assessment is necessary and therefore, it is highly recommendable that students can view their own presentations. Thus, the convenience of combining both strategies. As further work, the data regarding the use of the educational resources on the institutional learning platform will be analysed, together with the global learning achievement. And all that together with the evaluation of the project by students and teachers.

Keywords: German as a Foreign Language, effective oral communication, learning-oriented-assessment, interactive videos.

\section{INTRODUCCIÓN}

El interés por el aprendizaje de la lengua alemana en la Universitat Politècnica de València (UPV) ha sido constante desde su implantación en los antiguos planes de estudio. Sin embargo, en los últimos años este interés se ha incrementado al evidenciarse una importante oferta laboral para graduados en Ingeniería en Alemania. El alumnado de la UPV dirige su atención a este país también para especializar sus estudios realizando estancias Erasmus+, másteres y prácticas en empresas. Una de las mayores dificultades de adaptación es la lengua.

Además, es indudable que, tanto en el ámbito académico como en el profesional, la capacidad de realizar presentaciones orales es necesaria, tal y como lo indican los estudios de Minks (2006) o Marks (2015), entre otros, que resaltan su importancia y señalan esta capacidad de comunicar como "punto débil" entre los estudiantes en general.

Esta es la razón fundamental de la inclusión de la comunicación oral efectiva en nuestras aulas, pero tanto para desarrollarla, como para mejorarla, es imprescindible que el alumnado sea consciente de su proceso de aprendizaje (Nunan, 1988). En este proceso, la evaluación formativa (Black \& William, 1998) y la autoevaluación juegan un papel fundamental, desde el momento en que ambas activan la reflexión sobre los avances en el aprendizaje: con la valoración por parte del docente en el primer caso y con la valoración del propio estudiante en el segundo.

Por otra parte, es tal la importancia del vídeo en la sociedad actual, que está transformando la forma en que nos comunicamos, ocupamos nuestro tiempo de ocio, colaboramos y, por supuesto, aprendemos. En el ámbito educativo, el vídeo es un elemento fundamental en muchas de las nuevas tendencias, tales como en enseñanza online e híbrida, MOOC o modelos de Clase Inversa (Kaltura, 2016). Desde hace tiempo, nuestra Universidad apostó por la producción masiva de recursos didácticos en vídeo (Turró, Cañero \& Busquets, 2011), la grabación en vídeo de las clases presenciales (Turró, Cañero \& Busquets, 2014) o, más recientemente, la inclusión de vídeos como elemento habitual dentro de los MOOC. 
Dado que en las asignaturas de Alemán se vienen grabando las presentaciones orales de los estudiantes desde el curso 2014/15, parece conveniente incorporar estos vídeos como material propio para los alumnos que cursan estas asignaturas, con el fin de que puedan apreciar la aptitud de sus pares y así ellos se sientan también capaces. Este aspecto es básico, puesto que la mayor parte del alumnado se encuentra en un nivel de iniciación en el aprendizaje de la lengua alemana. En trabajos anteriores, como el de Forés \& Gil (2004), ya se comprobó la viabilidad de introducir las presentaciones orales también en niveles de iniciación.

Tal y como apunta Kolås (2015), la utilización de lo que se conoce como vídeos interactivos puede ser una estrategia metodológica muy apropiada, puesto que permite interrumpir la reproducción para insertar comentarios, preguntas, enlaces a otros recursos, etc. con el fin de resaltar aquello que los docentes consideren más relevante.

Una revisión de la literatura nos permite encontrar distintos trabajos que incorporan el uso de vídeos interactivos en el aprendizaje de lenguas extranjeras. Por ejemplo, Povey (2016) incluye los vídeos interactivos entre los recursos y herramientas más adecuados para diseñar actividades que trabajen la comprensión oral. Por su parte, Alvarado, Coelho \& Dougherty (2016) justifican la utilización de vídeos interactivos en el aprendizaje del inglés como lengua extranjera, porque apoyan distintos estilos de aprendizaje y favorecen el trabajo autónomo de los estudiantes. También ponen el foco en reforzar la comprensión oral haciendo uso de materiales auténticos y culturalmente apropiados. Por último, Bakla (2017) afirma que, pese a sus potenciales beneficios, los vídeos interactivos han sido escasamente utilizados en el ámbito del aprendizaje de lenguas. En su trabajo describe las posibilidades instructivas que supone añadir elementos interactivos a los vídeos, como la evaluación formativa, el estudio de vocabulario, o su uso como elementos iniciales en sesiones de discusión o modelos de Clase Inversa.

El trabajo continúa con la siguiente estructura: la sección 2 presenta el contexto y los objetivos del proyecto; la sección 3 describe la propuesta y justifica las decisiones tomadas; la sección 4 introduce los resultados y su correspondiente discusión y la sección 5 aborda las conclusiones y los trabajos futuros.

\section{CONTEXTO}

El proyecto está contextualizado en las asignaturas de Alemán A1, A2 y B1 (los tres primeros niveles del Marco Común Europeo de Referencia para las Lenguas Extranjeras). Estas asignaturas tienen carácter optativo dentro del Bloque de Formación Complementaria en muchos de los Grados de la UPV y son punto de control de las competencias transversales CT08 "Comunicación efectiva” y CT11 “Aprendizaje permanente".

La puesta en marcha de este proyecto permitirá elaborar recursos didácticos en soporte vídeo, creados en las mismas prácticas de las asignaturas involucradas, con el alumnado como protagonista y agente principal del proceso. Este material pasará a formar parte de los recursos docentes de las asignaturas, tanto en los grupos de Clase Inversa, como en el resto de grupos.
Con ayuda de los recursos generados, se espera un aprendizaje más eficaz de la competencia comunicativa al remarcar aquellos aspectos formales, lingüísticos y de contenido, que son necesarios para alcanzar un alto nivel de logro de la competencia.

En resumen, el objetivo principal es mejorar el conocimiento de la lengua mientras se desarrolla la competencia comunicativa.

Los objetivos específicos del proyecto son los siguientes:

- Grabar presentaciones orales realizadas por el alumnado de las asignaturas Alemán A1, A2 y B1, con el fin de generar un corpus oral.

- Clasificar las grabaciones por niveles de competencia lingüística y por aspectos a resaltar.

- Crear vídeos interactivos para mostrar ejemplos de buenas prácticas y errores comunes en el aprendizaje de la expresión oral y comunicación efectiva.

- Diseñar tareas de aprendizaje planificadas siguiendo el programa de la asignatura.

- Fomentar la reflexión del alumno sobre su aprendizaje comparando sus resultados con los de sus pares.

- Evaluar la competencia en la comunicación oral según los criterios definidos en las rúbricas.

\section{DESCRIPCIÓN}

Durante el curso 2015/16 se realizó un estudio preliminar de las presentaciones orales llevadas a cabo en dos grupos de la asignatura Alemán A1, el grupo A1A4 y A1A5 con un total de 44 alumnos. El análisis de los datos ha mostrado unos buenos resultados, tanto desde la percepción del alumnado, como desde el rendimiento académico. La autoevaluación fue igualmente fomentada durante ese curso y se recogieron evidencias positivas en este sentido. La práctica de exponer en público mejoró, ya que el alumnado ganó en seguridad y destreza (Gil-Salom \& Benlloch-Dualde, 2016).

Las estrategias metodológicas y de evaluación durante el curso 2016/17 se han mantenido, si bien no tenemos todavía el análisis de los resultados correspondientes. Para complementar la evaluación formativa y así poder mejorar los logros de aprendizaje de los estudiantes, se ha considerado conveniente la producción de una serie de vídeos interactivos a partir de las presentaciones orales de los estudiantes. Los elementos centrales a reforzar serían lógicamente aquellos que fueron indicados por los estudiantes como puntos débiles; entre estos últimos se encuentran los nervios, la pronunciación y el vocabulario (Gil-Salom \& Benlloch-Dualde, 2016).

Dado que las herramientas institucionales asociadas a la iniciativa OpenCast no incluyen, por el momento, el soporte necesario para producir vídeos interactivos, se ha decidido hacer uso de herramientas comerciales.

Entre las herramientas disponibles, se ha optado por EDpuzzle (https://edpuzzle.com/), pues es gratuita tanto para profesores como para estudiantes (tan solo algunas características "premium”, como el libro de calificaciones, tiene un coste para los centros educativos) e incluye la mayoría de las características necesarias para el proyecto. Gracias a su sencilla interfaz, permite incorporar, en cualquier instante de la secuencia de vídeo, comentarios y cuestiones, 
tanto de tipo test como de respuesta abierta. Adicionalmente, permite obtener informes sobre quién ha visionado los vídeos y sobre las respuestas a las preguntas incorporadas en los mismos.

La Figura 1 muestra un ejemplo de vídeo interactivo realizado con EDpuzzle. En la parte inferior del vídeo, sobre la línea de tiempo, se puede observar que aparecen marcados una serie de instantes con un icono de pregunta. La aplicación muestra de este modo aquellos puntos de control de la secuencia donde se obliga al estudiante a detener la visualización, para así realizar algún tipo de acción, bien sea leer un comentario o responder a una pregunta.

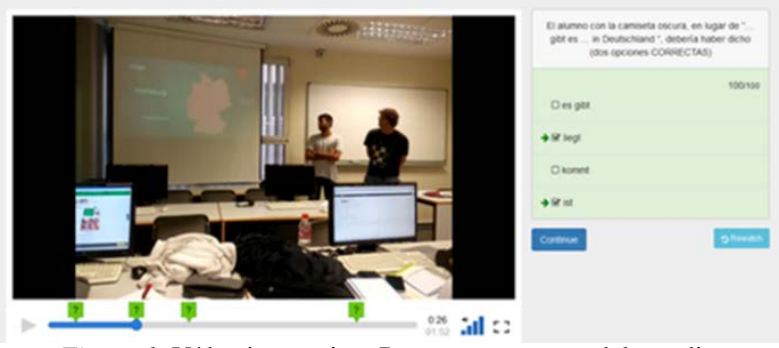

Figura 1: Vídeo interactivo. Respuesta correcta del estudiante.

En particular, como puede apreciarse en la imagen, en la segunda parada se incorpora una pregunta sobre el correcto uso de la lengua. En este caso, la aplicación muestra que las respuestas del estudiante han sido correctas.

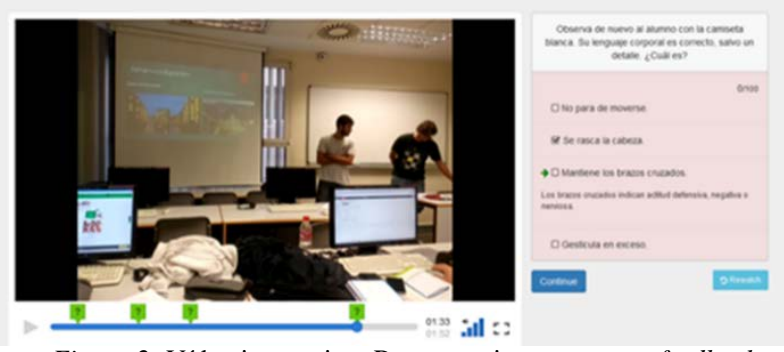

Figura 2: Vídeo interactivo. Respuesta incorrecta con feedback.

En la Figura 2 se observa que la secuencia se ha detenido en el último punto de control y que, en esta ocasión, la pregunta trata sobre el correcto uso del lenguaje corporal. Como la respuesta del estudiante ha sido incorrecta, la aplicación proporciona la respuesta adecuada, junto con la consiguiente explicación a modo de feedback. Se ofrece además la posibilidad de volver a visualizar (Rewatch) o continuar (Continue).

Siguiendo este enfoque es posible, por tanto, ofrecer al alumnado, en un formato familiar y atractivo, ejemplos de aquellos aspectos que domina y en los que puede reafirmar sus habilidades, así como de aquellos otros en los cuales se siente inseguro y puede mejorar.

\section{Resultados}

Con el fin de evaluar la utilidad de los vídeos interactivos se ha desarrollado un primer vídeo de ejemplo y se ha elaborado un cuestionario que han respondido, a modo de experiencia piloto, tanto estudiantes como profesores. Entre los primeros han participado alumnos matriculados en el presente curso 2016-17 en los grupos de Alemán 2 del segundo cuatrimestre, así como algunos alumnos que cursaron Alemán 1 el cuatrimestre anterior. El cuestionario ha sido presentado también a un conjunto de profesores escogidos, bien por participar en proyectos de innovación educativa o por ser docentes del departamento de Lingüística Aplicada, todos ellos de nuestra universidad. Un total de 10 estudiantes y otros tantos profesores han participado en la valoración.

El cuestionario incluye cinco preguntas de valoración en una escala Likert (Likert, 1974) de cinco puntos (Q1-Q3 y Q5Q6) para que los encuestados manifiesten su grado de acuerdo o desacuerdo (desde totalmente de acuerdo TA, hasta totalmente en desacuerdo, TD) sobre las posibilidades tanto de los vídeos interactivos en general, como de la herramienta utilizada en su elaboración; una pregunta de respuesta múltiple (Q4) para conocer, de entre una lista, qué aspectos podrían mejorar este tipo de recursos $\mathrm{y}$, por último, tres preguntas de respuesta abierta (Q7-Q9) con el fin de recoger aspectos positivos, aspectos negativos y sugerencias de mejora. A continuación, se muestran los enunciados de las distintas preguntas:

- Q1. Añadir comentarios y preguntas a las grabaciones aporta un valor añadido al vídeo como recurso didáctico.

- Q2. Disponer de vídeos interactivos que muestren errores frecuentes relacionados con el uso de la lengua alemana sería útil para la preparación de las presentaciones orales.

- Q3. Disponer de vídeos interactivos que muestren ejemplos de un uso correcto de la lengua alemana sería útil para la preparación de las presentaciones orales.

- Q4. Disponer de un conjunto variado de vídeos interactivos para preparar las presentaciones orales me ayudaría a mejorar en los siguientes aspectos: Estructura de la presentación / Nervios / Pronunciación / Vocabulario / Estructuras lingüísticas / Fluidez.

- Q5. La herramienta EDpuzzle es fácil de usar.

- Q6. Los vídeos interactivos podrían ayudarme a mejorar las presentaciones orales en general.

- Q7. ¿Qué aspectos positivos destacarías de los vídeos interactivos?

- Q8. ¿Qué aspectos negativos destacarías de los vídeos interactivos?

- Q9. ¿Alguna sugerencia de mejora respecto de los vídeos interactivos?

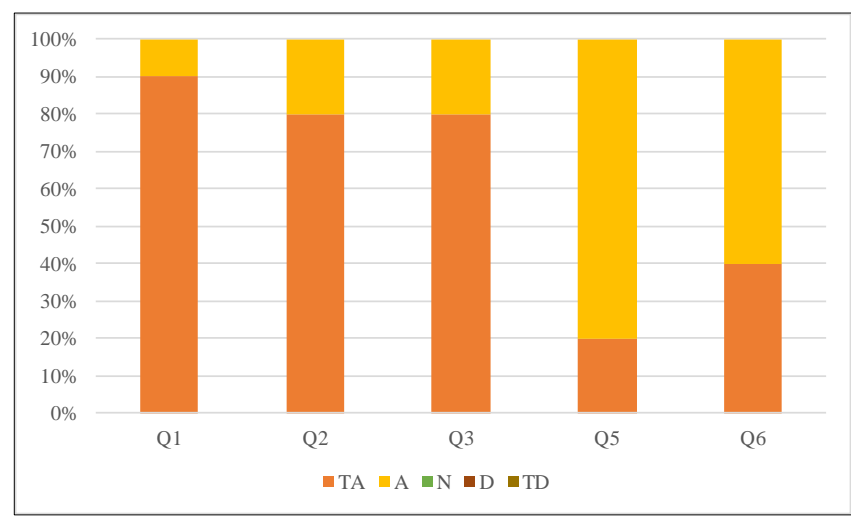

Figura 3: Respuestas de los estudiantes $(\mathrm{n}=10)$. 
En la Figura 3 se observa una muy buena valoración por parte de los estudiantes del concepto de vídeo interactivo (Q1) y de su utilidad, tanto para mostrar ejemplos de uso correcto de la lengua (Q2) como errores frecuentes (Q3). Sin embargo, son más críticos a la hora de valorar su utilidad para preparar presentaciones en general (Q6), lo que puede resultar curioso. La cuestión sobre la facilidad de uso de la herramienta EDpuzzle (Q5) es la peor valorada de estas cinco, si bien el $100 \%$ de los estudiantes responden positivamente (TA o A).

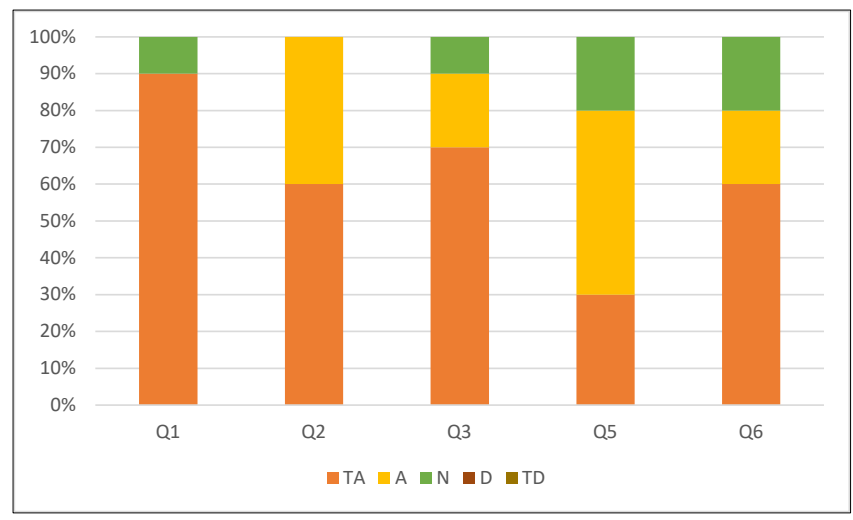

Figura 4: Respuestas de los profesores $(\mathrm{n}=10)$.

Los profesores, en general, también se sienten bastante positivos respecto de este tipo de recursos y su utilidad (Figura 4). En este caso, además, valoran mejor la utilidad de los vídeos interactivos para las presentaciones orales en general (un 60\% está totalmente de acuerdo, frente a un $40 \%$ de los estudiantes). También hay que notar varias respuestas neutras en diferentes cuestiones, en particular respecto a la herramienta de producción, lo que indica el escepticismo de algún profesor por este tipo de recursos.

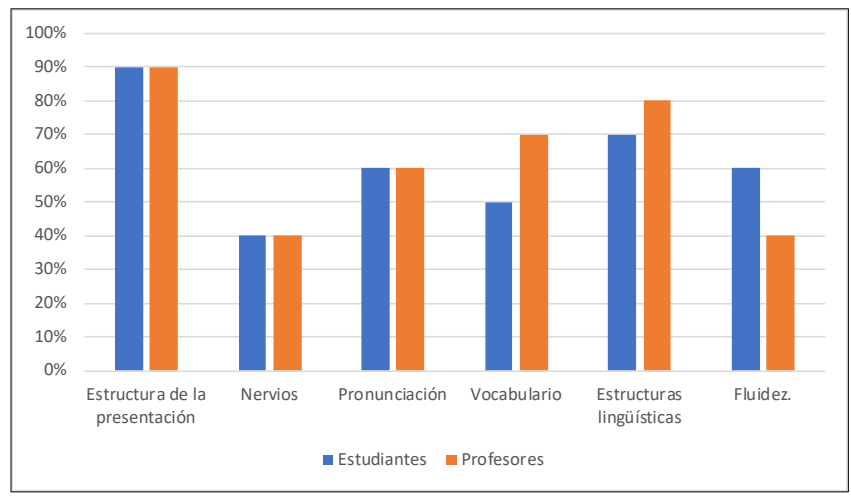

Figura 5: Respuestas a la Q4. Aspectos de mejora.

En la Figura 5, donde se resumen aquellos aspectos que podrían mejorar por el uso de los vídeos interactivos (Q4), se observa un gran paralelismo entre las respuestas de los estudiantes y profesores. La mayor diferencia se da en el caso de la fluidez y es tan solo del $20 \%$, siendo idéntico para la estructura de la presentación, el mejor valorado (90\%), la pronunciación y los nervios, siendo este el aspecto menos considerado.

En cuanto a las cuestiones de respuesta abierta, de los 18 comentarios que señalan aspectos positivos, cuatro de ellos hacen referencia al aprendizaje inter pares y cinco destacan la posibilidad de fijar la atención sobre aspectos de interés. En cuanto a los aspectos negativos, de los 17 comentarios, tres indican la necesidad o conveniencia de comentar aspectos con el profesor y con los compañeros, además de visualizar los vídeos interactivos. En dos ocasiones se dice que no hay ningún aspecto negativo. En relación a las sugerencias de mejora, de los 14 comentarios, en cuatro ocasiones se sugiere que hay que mejorar el sonido, mientras que en dos ocasiones se comenta que habría que indicar el tema que se desea remarcar (lenguaje verbal, corporal, estructura general de la presentación, etc.).

Para terminar este apartado, se han seleccionado aquellas respuestas, tanto de estudiantes como profesores, que responden en mayor grado a los objetivos iniciales de nuestro trabajo. Entre las de los estudiantes:

- $\quad$ Pienso que es una buena idea para aprender cómo realizar exposiciones orales y cómo no. Siempre buscamos ejemplos en los que fijarnos y si además se explican los errores pienso que puede ser muy útil.

- Me ha gustado que sean ejemplos de compañeros, porque me permite comprobar el nivel que yo debería tener en mi contexto. La inclusión de preguntas hace que se produzca un diálogo, tenga que estar más atento $y$, sobre todo, que no desconecte.

Entre las respuestas de los profesores, cabe citar:

- Permiten aportar información valiosa sobre aspectos significativos para el aprendizaje. Potencian mucho la utilidad y la significación del vídeo. Permiten realimentación al profesor.

- Presentaría a los alumnos vídeos específicos de errores o aciertos. Por ejemplo, unos donde se trabajara solo el lenguaje corporal, otro de lengua o pronunciación, otro de pobreza o riqueza de vocabulario, otro de estructuración, etc. $Y$, solo después de trabajarlos de este modo, presentaría uno completo (es decir, trabajar el proceso).

En términos generales, tanto los aspectos negativos señalados en los comentarios, como las sugerencias de mejora, resultan abordables en el ámbito del proyecto, por lo que creemos pueden contribuir a mejorar la utilidad de los próximos recursos producidos.

\section{CONCLUSIONES}

Los objetivos de este trabajo persiguen unas metas concretas y propias de nuestro contexto profesional. Por un lado, el aprendizaje de la lengua alemana, que cuenta con una larga presencia en la UPV para complementar el perfil de los estudiantes de cara a su futuro, tiene la oportunidad de ver mejoradas sus herramientas educativas y actualizar, de este modo, su metodología didáctica. El análisis y valoración de la evaluación formativa como esencia del proceso de aprendizaje, se ve igualmente favorecido con la propuesta, al poder estudiar en detalle las características individuales y grupales de nuestros estudiantes. Además, estamos convencidos del valor añadido que unos vídeos interactivos bien diseñados puede representar en los logros del aprendizaje de los estudiantes. Por último, este enfoque puede facilitar el desarrollo y evaluación de las competencias transversales "Comunicación efectiva” y “Aprendizaje permanente”, 
competencias indispensables en la formación de los estudiantes, especialmente en la de lenguas extranjeras.

Por último, considerando la utilización de equipamiento doméstico para las grabaciones, la sencillez de los procesos de producción de los vídeos interactivos y el uso de herramientas software gratuitas, pensamos que el enfoque propuesto es sostenible y la curva de aprendizaje para elaborar este tipo de recursos, razonable. Por todo ello y, analizadas las respuestas al cuestionario, pensamos que la propuesta, con las adaptaciones pertinentes, puede ser también de aplicación en otras asignaturas o ámbitos bien diferentes al descrito en este trabajo.

Como trabajo futuro, en la línea de lo que se conoce como Analítica del Aprendizaje o Learning Analytics (Long \& Siemens, 2011), parece conveniente diseñar una serie de informes específicos que permitan analizar los accesos del alumnado a los diferentes recursos didácticos utilizados en la propuesta, así como otros estudios que los relacionen con el nivel de logro en el aprendizaje del alumnado. Del mismo modo, la utilización de estrategias de investigación cualitativa para el análisis de cuestionarios o entrevistas a los participantes, puede contribuir a una mejor evaluación del proyecto.

\section{AGRADECIMIENTOS}

Este trabajo se ha realizado en el marco del Proyecto de Innovación y Mejora Educativa PIME/2016/A/019, financiado por el Vicerrectorado de Estudios, Calidad y Acreditación de la Universitat Politècnica de València. También ha contado con la ayuda de la Escuela Técnica Superior de Ingeniería Informática (ETSINF) de la UPV.

\section{REFERENCIAS}

Alvarado, N. C., Coelho, D., \& Dougherty, E. (2016). Mobile apps for ELLs: Supporting language learning with engaging digital tools. Argentinian Journal of Applied Linguistics, 4 (1), 43-58.

Bakla, A. (2017). Interactive Videos in Foreign Language Instruction: A New Gadget in Your Toolbox* Yabanc1 Dil Eğitiminde Etkileșimli Videolar: Takım Çantanızda Yeni Bir Alet. Mersin University Journal of the Faculty of Education, 13 (1), 124-137.

Black, P. \& William, D. (1998). Inside the black box: Raising standards through classroom assessment. Phi Delta Kappan, $\quad 80 \quad$ (2), $139-148$. https://doi.org/10.1177/003172171009200119

Forés López, M.L. \& Gil Salom, D. (2004). Mündliche Präsentationen (Referatstechnik). In M.J. Domínguez, B. Lübke and A. Mallo, (Eds), El alemán en su contexto español = Deutsch im spanischen Kontext; Actas del IV Congreso de la Federación de Asociaciones de Germanistas y Profesores de Alemán en España. Santiago de Compostela, Spain, 26-28 September 2002 (pp. 309-316). Santiago de Compostela: Universidade de Santiago de Compostela.

Gil-Salom, D., \& Benlloch-Dualde, J.V. (2016). Student assessment of oral presentations in German as a Foreign Language. Procedia - Social and Behavioral Sciences, 228: 656-661. https://doi.org/10.1016/j.sbspro.2016.07.100

Kaltura, Inc. (2016), The State of Video in Education 2016: A Kaltura Report. Retrieved from https://corp.kaltura.com/sites/default/files/The\%20State \%20of\%20Video\%20in\%20Education\%202016\%20\%20A\%20Kaltura\%20Report.pdf?aliId=165316164

Kolås, L. (2015). Application of interactive videos in education. In 2015 International Conference on Information Technology Based Higher Education and Training (ITHET), Lisboa, Portugal, 11-13 June 2015 (pp. 1-6). Los Alamitos, CA: IEEE Computer Society. https://doi.org/10.1109/ITHET.2015.7218037

Likert, R. (1974). A method of constructing an attitude scale. In G. Maranell (Ed.), Scaling; A Sourcebook for Behavioral Scientists (pp. 233-243). Chicago, IL: Aldine.

Long P. \& Siemens, G. (2011). Penetrating the Fog: Analytics in Learning and Education. EDUCAUSE Review, 46 (5), 31-40.

Marks, D. (2015). Prüfen sprachlicher Kompetenzen internationaler Studienanfänger an deutschen Hochschulen - Was leistet der TestDaF? Zeitschrift für Interkulturellen Fremdsprachenunterricht, 20 (1), 21-39. Retrieved from http:/tujournals.ulb.tudarmstadt.de/index.php/zif/

Minks, K-H. (2006). Kompetenzen für den Arbeitsmarkt: Was wird vermittelt? Was wird vermisst? Retrieved from: http://www.dzhw.eu/pdf/pub_vt/22/2006_07_28_Vortra g_Minks_Siemensforum.pdf

Nunan, D. (1988). The Learner-Centred Curriculum. Cambridge: Cambridge University Press.

Povey, E. (2016). Extensive Listening: Pedagogy, Resources, and Tools. International Journal of Educational Investigations, 3 (7), 35-49.

Turró, C., Cañero, A. \& Busquets, J. (2011). Video learning objects creation with polymedia. In 2010 IEEE International Symposium on Multimedia (ISM), (pp. 371376). Los Alamitos, CA: IEEE Computer Society. https://doi.org/10.1109/ISM.2010.69

Turró, C., Cañero, A. \& Busquets, J. (2014). Grabación automatizada de clases magistrales: el proyecto Videoapuntes de la UPV. Revista de Educación a Distancia, 40, 371-376. 